\title{
Collisional and Radiative Processes in Adiabatic Deceleration, Deflection, and Off-Axis Trapping of a Rydberg Atom Beam
}

\author{
Ch. Seiler, S. D. Hogan, H. Schmutz, J. A. Agner, and F. Merkt \\ Laboratorium für Physikalische Chemie, ETH Zürich, CH-8093 Zürich, Switzerland
}

(Received 9 December 2010; published 17 February 2011)

\begin{abstract}
A supersonic beam of Rydberg hydrogen atoms has been adiabatically deflected by $90^{\circ}$, decelerated to zero velocity in less than $25 \mu \mathrm{s}$, and loaded into an electric trap. The deflection has allowed the suppression of collisions with atoms in the trailing part of the gas pulse. The processes leading to trap losses, i.e., fluorescence to the ground state, and transitions and ionization induced by blackbody radiation have been monitored over several milliseconds and quantitatively analyzed.
\end{abstract}

DOI: 10.1103/PhysRevLett.106.073003

PACS numbers: $32.60 .+\mathrm{i}, 32.80 . \mathrm{Ee}$

The use of cold atoms and molecules in spectroscopic experiments and in studies of bimolecular collisions at low temperature has recently stimulated considerable efforts to devise experimental tools capable of producing stateselected cold molecular samples, either in electromagnetic traps or in velocity-controlled gas beams [1-4]. The use of supersonic beams in combination with deceleration represents one of the main approaches to generate cold gasphase samples [3]. Deceleration methods enable one to produce cold samples of polar molecules by multistage Stark deceleration [5], of paramagnetic atoms and molecules by multistage Zeeman deceleration [6-8], and, at least in principle, of any atom or molecule by RydbergStark [9-11] and optical deceleration [12]. These methods and variants based on traveling electric traps have been developed recently $[10,13]$, and further approaches, including the use of microfabricated devices [14] and continuous trap-loading schemes [15], are promising for many applications in chemical physics, high-resolution spectroscopy, metrology, quantum information processing, and antihydrogen studies.

Using these methods, the atoms or molecules to be decelerated only represent a small fraction of the gas in the supersonic expansion, the largest fraction being the carrier gas (usually a rare gas), precursor molecules (e.g., $\mathrm{N}_{3} \mathrm{H}$ for $\mathrm{NH}$ [15]), and molecules in quantum states not suitable for deceleration. As the deceleration proceeds, the increasing velocity difference between decelerated and undecelerated particles renders collisions unavoidable, potentially leading to loss and heating of the decelerated particles. These undesirable processes, referred to as the carrier-gas problem below, are difficult to quantify and rarely mentioned in the context of deceleration experiments. The carrier-gas problem becomes acute when (1) the deceleration is strong and a large velocity slip builds up before the trailing part of the gas pulse has overtaken the decelerated molecules, (2) the decelerated molecules are directed toward an on-axis target, such as a surface or another gas sample, because the impact of the carrier gas on the target may alter its properties or mask the effect one desires to study, and (3) cumulative trap loading from successive gas pulses is attempted, because the cold molecules trapped in a given experimental cycle are ejected by the carrier gas in subsequent pulses. The carrier-gas problem represents a limitation in our Rydberg-Stark deceleration and trapping experiments on atomic and molecular hydrogen $[11,16]$, and has so far prevented us from disentangling the effects of collisional and radiative trap-loss processes.

We present here a Rydberg-Stark deceleration scheme which enables the rapid, adiabatic, $90^{\circ}$ deflection of the Rydberg sample followed by the loading of an off-axis trap. By comparing the results of on-axis and off-axis trapping experiments at room temperature and at $125 \mathrm{~K}$, we have (1) quantified important aspects of the carrier-gas problem in Rydberg-Stark deceleration, (2) studied the loss of Rydberg atoms from the trap under conditions where it is dominated by radiative processes, (3) clarified the role of fluorescence and blackbody-radiation-induced transitions, and (4) made first measurements of trap losses resulting from the zero-field region near the trap center.

The Rydberg-Stark decelerator and off-axis trap is presented in Fig. 1(a). A supersonic beam of hydrogen atoms [dashed arrow in Fig. 1(a)] is produced by laser photolysis of $\mathrm{NH}_{3}$ seeded in krypton [17]. The beam moves in the positive $z$ direction, passes through a skimmer, and enters the electrode setup which forms the Rydberg-Stark decelerator and trap. Photoexcitation to selected Rydberg-Stark states with principal quantum number $n$ around 30 is achieved by resonant two-photon excitation via the $2^{2} \mathrm{P}$ state. The two laser beams at 121 and $\sim 365 \mathrm{~nm}$ are counterpropagating, enter the excitation region through a set of 1.6-mm-diameter holes in electrodes 1,2 , and 7, and cross the $\mathrm{H}$ atom beam at right angles. Following photoexcitation, a series of pulsed potentials are applied to the electrodes to decelerate and load the Rydberg atoms into the off-axis trap located $6 \mathrm{~mm}$ away from the undeflected supersonic beam.

The sequence of pulsed voltages applied to the ten electrodes can be designed so as to either trap the atoms 


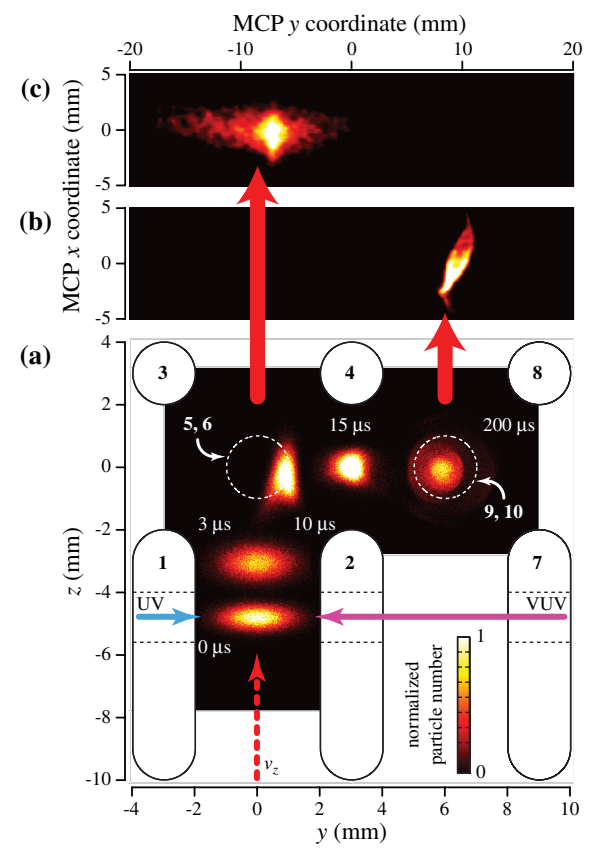

FIG. 1 (color online). (a) Schematic diagram of the RydbergStark deceleration, deflection, and trapping device, with simulated snapshots of a $n=30, k=19$ Rydberg $\mathrm{H}$ atom cloud at the time of photoexcitation $(t=0 \mu \mathrm{s})$ and at times $3,10,15$, and $200 \mu \mathrm{s}$. The electrodes are numbered 1-10 and electrodes 5 (6) and 9 (10) are located above (below) the plane of the figure. (b), (c) Experimental images of $\mathrm{H}^{+}$ions extracted from the off-axis (b) and on-axis (c) traps. VUV is vacuum ultraviolet.

on axis between electrodes $1-4$ as demonstrated in Ref. [16] or off axis between electrodes 2, 4, 7, and 8 [see Fig. 2(a) for the sequence used in this work]. In the photoexcitation and trapping configuration [Fig. 2(b)], potentials of $+12 \mathrm{~V}(-12 \mathrm{~V})$ are applied to electrodes 1,4 , and 7 (2,3, and 8$)$ forming two electric quadrupole traps in the $y z$ plane, with minima located at the midpoint of the four electrodes 1-4 (on-axis trap) and 2, 4, 7, 8 (off-axis trap). Electrodes 5 and 9 (6 and 10) are separated by $10 \mathrm{~mm}$ in the $x$ direction and are operated at potentials of $-22 \mathrm{~V}$ $(+22 \mathrm{~V})$ to achieve confinement of the Rydberg atom cloud in the $x$ direction and generate a field of $10 \mathrm{~V} / \mathrm{cm}$ at the trap minimum. The lowest saddle point in the electric-field distribution is $46 \mathrm{~V} / \mathrm{cm}$, which corresponds to a trap depth of $1.3 \mathrm{~cm}^{-1}$ (or $E / k_{B}=1.9 \mathrm{~K}$ ) for the $n=30, k=19$ Rydberg atoms used in the experiments. The initial density and temperature of the trapped atoms are estimated to be $10^{6} \mathrm{~cm}^{-3}$ and $150 \mathrm{mK}$ using the methods described in Ref. [16].

To study the effects of blackbody radiation, the electrode setup was enclosed on five sides by a $90 \mathrm{~mm} \times 80 \mathrm{~mm} \times$ $60 \mathrm{~mm}$ copper heat shield and cooled to $\sim 125 \mathrm{~K}$ using a liquid-nitrogen thermal bath. The Rydberg atoms loaded into the on-axis (or off-axis) trap are detected by pulsed field ionization (PFI) by applying a potential of $+1.25 \mathrm{kV}$ to electrodes 1 and 2 (or 2 and 7) which extracts the $\mathrm{H}^{+}$ions toward a microchannel-plate (MCP) detector

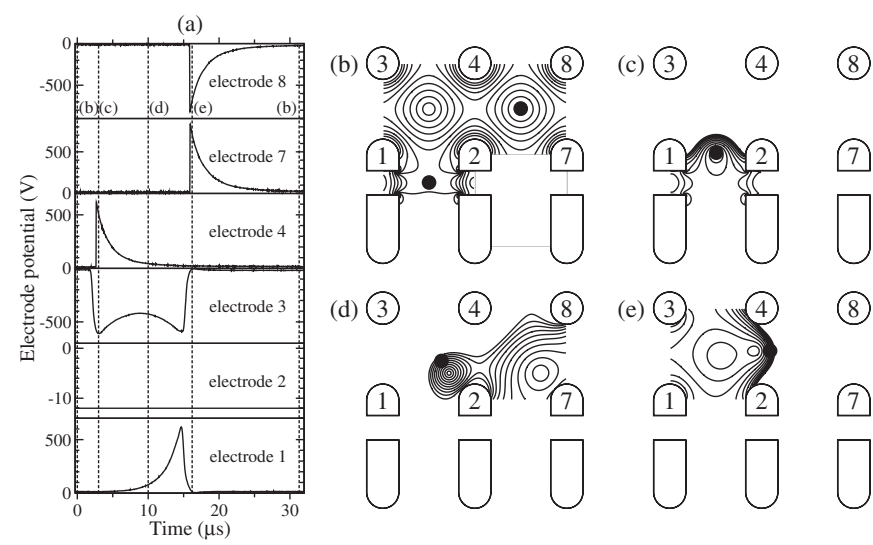

FIG. 2. (a) Off-axis-trapping voltage pulse sequence. (b)(e) Schematic diagram of the electrode setup in the $y z$ plane at the time of photoexcitation and after completion of the trapping process (b), in the initial phase of the on-axis deceleration (c), during the $90^{\circ}$ deflection process (d), and the final deceleration (e). The lines of constant electric fields range from 10 to $100 \mathrm{~V} / \mathrm{cm}$ in steps of $10 \mathrm{~V} / \mathrm{cm}$ (b), and from 20 to $200 \mathrm{~V} / \mathrm{cm}$ in steps of $20 \mathrm{~V} / \mathrm{cm}$ in (c)-(e). The dots indicate the center of the Rydberg atom cloud.

connected to a phosphor screen. Images of the $\mathrm{H}^{+}$ions are recorded with a CCD camera. The ion optics were designed so that atoms field ionized in the on- and offaxis traps are easily distinguished [Figs. 1(b) and 1(c)]. Numerical particle-trajectory simulations were carried out as described in Ref. [18] to predict and analyze the experimental results.

Figures 1(b) and 1(c) display images of the $\mathrm{H}^{+}$ions recorded following deceleration of a $600 \mathrm{~m} / \mathrm{s}$ beam of $n=30, k=19$ atoms. To obtain the image in Fig. 1(c), the atoms were extracted from the position of the on-axis trap, whereas to obtain the image in Fig. 1(b), the atoms were first deflected by $90^{\circ}$ before being loaded into the offaxis trap. In the former (latter) case, the entire trapping process took less than $10 \mu \mathrm{s}(25 \mu \mathrm{s})$. The asymmetric shape of the $\mathrm{H}^{+}$image in Fig. 1(b) results from the details of the electrode and ion optics configuration. The removal of the Rydberg atoms from the beam axis in only a few microseconds followed by off-axis trapping enables us to study the trap decay dynamics without interference from collisional processes.

The operational principle of the trap and its main characteristics are described in Figs. 2 and 3. The electric potentials applied to the relevant electrodes during deceleration and trap loading are presented in Fig. 2(a). The pulse sequence consists of five phases: photoexcitation in the trap configuration $(t=0 \mu \mathrm{s})$, initial deceleration and deflection $(0<t<5 \mu \mathrm{s})$, turning of the electric-field minimum around electrode $2(5 \mu \mathrm{s}<t<15 \mu \mathrm{s})$, final deceleration in the $y$ direction $(15 \mu \mathrm{s}<t<25 \mu \mathrm{s})$, and off-axis trapping ( $t \geq 25 \mu \mathrm{s})$. The electric-field distributions in the $y z$ plane at the beginning of each of these five phases are displayed in Figs. 2(b)-2(e). The corresponding times are indicated by dashed lines in Fig. 2(a). 

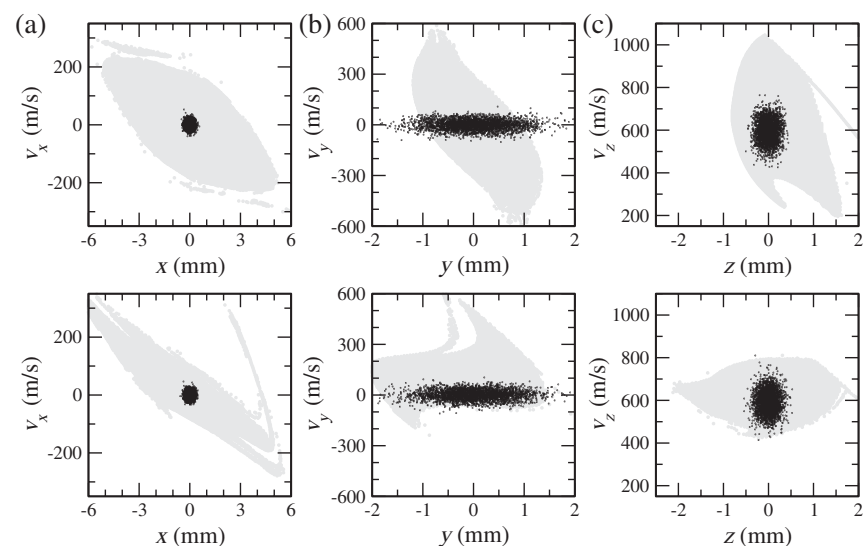

FIG. 3. (a)-(c) Simulated phase-space acceptance (gray areas) of the electrostatic deceleration and on-axis (top panels) and offaxis (bottom panels) trapping procedures for $n=30, k=19$ Rydberg states of $\mathrm{H}$ in the (a) $x$, (b) $y$, and (c) $z$ directions. The photoexcited Rydberg atoms are shown as black dots.

The gray areas in Figs. 3(a)-3(c) represent the calculated phase-space acceptance of the deceleration and trapping process in the $x, y$, and $z$ dimensions, respectively. The top (bottom) panels correspond to on-axis (off-axis) trapping. For the simulations, we adopted the mean longitudinal velocity $(600 \mathrm{~m} / \mathrm{s})$ and the velocity and position distributions defined by the geometric constraints of the experiment (see Ref. [18]). In the phase-space diagrams, the photoexcited atoms are represented as black dots. The figure demonstrates that $\sim 90 \%$ of the initially prepared Rydberg atoms end up in the trap. Most of the loss occurs in the $y$ dimension.

In all experiments described below, the trap decay rates were measured by monitoring the $\mathrm{H}^{+}$ion signal as a function of the delay time between photoexcitation and PFI. Figure 4(a) shows measurements of the trap decay at room temperature following on-axis trapping (open circles) and off-axis trapping (full circles) of low-field-seeking $n=30$ Rydberg states of $\mathrm{H}$. The decay rates are identical beyond $170 \mu \mathrm{s}$, and the difference at early times is evidence for collisional losses of $\approx 40 \%$ induced by the trailing part of the gas pulse and of their suppression by the off-axis trapping procedure. The fluorescence lifetimes $\tau_{\mathrm{fl}}$ of a $n=30$ Rydberg-Stark state can be estimated using Table 15 of Bethe and Salpeter and applying the expected $n^{4.36}$ scaling law [19] to be $220 \mu$ s and would correspond to the decay described by the dotted line. Decay channels other than fluorescence therefore play a role in the experiment. Their time constant can be determined to be $\tau=$ $350 \mu \mathrm{s}$ using $1 / \tau_{\mathrm{obs}}=1 / \tau_{\mathrm{fl}}+1 / \tau$, with $\tau_{\mathrm{obs}}=135 \mu \mathrm{s}$. The additional decay is attributed to blackbody radiation effects, but contributions from Rydberg-Rydberg collisions cannot be excluded at early times. Transitions between neighboring Rydberg states induced by blackbody radiation are such that they do not change the dipole moment significantly, and do not lead to trap losses but to a gradual redistribution of the initial population to neighboring Rydberg states. At room temperature, this redistribution rendered the observed trapping times largely independent of the $n$ value of the initially prepared Rydberg states. The observed trap loss can be attributed to direct ionization by blackbody radiation, if it is assumed that approximately $8 \%$ of the expected blackbody-radiation-induced depopulation of the initial state leads to ionization, as expected on the basis of the results presented in Refs. [20-22]. A further indication of the importance of blackbody-radiationinduced ionization was the observation that $\tau$ was reduced by a factor of $\sim 3$ to $\approx 110 \mu$ s after introduction of the copper heat shield under otherwise identical conditions (i.e., $T=300 \mathrm{~K}$ ). The low-frequency radiation field is indeed strongly influenced by the mode structure resulting from metallic surfaces surrounding the experimental volume.

$n$-dependent trap decay becomes observable after cooling the device to $\sim 125 \mathrm{~K}$, as illustrated by the
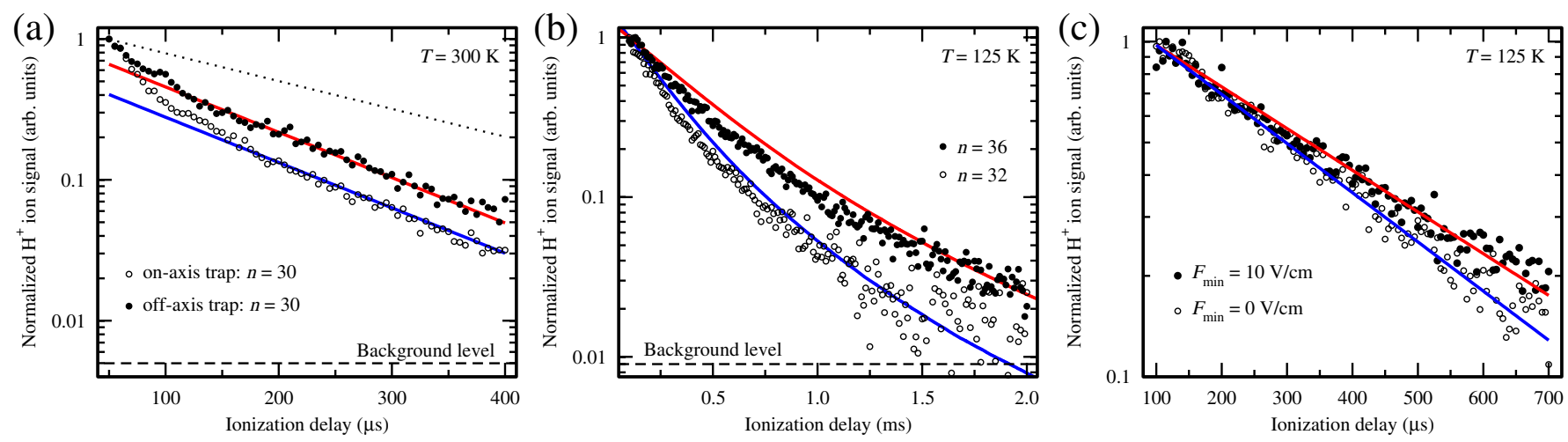

FIG. 4 (color online). (a) On-axis (open circles) and off-axis (full circles) trap loss of initially prepared $n=30, k=19$ Stark states. The dotted line corresponds to the $n=30, k=19$ radiative lifetime. The solid lines are single-exponential functions, fitted to the experimental data beyond $200 \mu \mathrm{s}$. (b) Measurement of the decay of atoms from the off-axis trap for $n=32$ and $n=36$ hydrogen Rydberg states at an electrode temperature of $125 \mathrm{~K}$ up to $2 \mathrm{~ms}$ with the corresponding Monte Carlo simulations. (c) $\mathrm{H}^{+}$ion signal detected from the off-axis electrostatic trap as a function of the delay between photoexcitation to the $n=36$ Rydberg-Stark state and PFI for traps with a zero field minimum (open circles) and a $10 \mathrm{~V} / \mathrm{cm}$ field minimum (full circles). The solid lines represent fitted single-exponential functions. 
measurements presented in Fig. 4(b) which were carried out following excitation to $n=32, k=17$ and $n=36, k=15$ Rydberg states. The trap-loss curves in Fig. 4(b) show a nonexponential decay that was modeled by a Monte Carlo simulation based on the following assumptions. (1) Trap loss is caused either by fluorescence to the ground state $\left(\tau_{\mathrm{fl}}\right.$, see above), by ionization induced by low-frequency radiation $\left(\tau_{\mathrm{I}}\right)$, or by multiple transitions to Rydberg states with $n$ values outside the range $(n=24-60)$ that can be trapped. (2) Transition rates $K_{n k, n^{\prime} k^{\prime}}$ to neighboring Rydberg states with $\left|n-n^{\prime}\right| \leq 5$ and $n k=n^{\prime} k^{\prime}$ are dominant and can be calculated from the Einstein $A$ coefficient and the photon occupation number $\bar{n}$ with (in $\mathrm{s}^{-1}$ )

$$
K_{n k, n^{\prime} k^{\prime}}=\bar{n} \frac{64 \pi^{4} \omega_{n k, n^{\prime} k^{\prime}}^{3}}{3 h c^{3} \times 4 \pi \varepsilon_{0}}\left|\left\langle n k m=0\left|\mu_{z}\right| n^{\prime} k^{\prime} m^{\prime}=0\right\rangle\right|^{2},
$$

using the Stark wave functions in parabolic coordinates from Ref. [23]. (3) The ionization rate $1 / \tau_{\text {I }}$ corresponds to $8 \%(4 \%)$ of the total blackbody-radiation-induced depopulation rate $1 / \tau_{\mathrm{BB}}^{\text {tot }}$ at $300 \mathrm{~K}(125 \mathrm{~K})$ as expected from Ref. [24]. (4) This rate can be estimated from the sum $1 / \tau_{\mathrm{BB}}^{\Delta n}$ of the rates of all transitions with $|\Delta n| \leq 5$ using $1 / \tau_{\mathrm{BB}}^{\mathrm{tot}}=1 / \tau_{\mathrm{BB}}^{\Delta n}+1 / \tau_{\mathrm{I}}$. This model accounts well for the experimental observations, however, only if the occupation number $\bar{n}$ is raised by a factor of $\approx 3$, which we believe is also caused by the copper heat shield surrounding the device. Our calculations [solid lines in Fig. 4(b)] predict that fluorescence to the ground state, ionization, and multiple $\Delta n$ transitions to untrappable Rydberg states account for $87 \%, 12 \%$, and $1 \%$, respectively, of the observed trap loss during the first $2 \mathrm{~ms}$ at $125 \mathrm{~K}$. At $300 \mathrm{~K}$, these percentages are $57 \%, 40 \%$, and $3 \%$, respectively. This prediction implies that continuous loading of ground state $\mathrm{H}$ atoms into a magnetic trap following Rydberg-Stark deceleration should be possible and become more efficient as the temperature is reduced. These lifetime measurements are of importance in the interpretation of $\mathrm{H}$ atom photofragment translational spectra recorded using Rydberg tagging methods [25].

Figure 4(c) compares two measurements of trap decay following off-axis trapping of $n=30, k=19$ Rydberg atoms. The first was carried out with the trap minimum at $0 \mathrm{~V} / \mathrm{cm}$ (electrodes 9 and 10 both at $+22 \mathrm{~V}$, open circles), the second with the trap minimum at $10 \mathrm{~V} / \mathrm{cm}$ (electrodes 9 and 10 at $\pm 22 \mathrm{~V}$, full circles). In the former (latter) case, the decay up to $700 \mu \mathrm{s}$ is fitted by an exponential function with a time constant of $300 \mu \mathrm{s}$ $(350 \mu \mathrm{s})$. This measurement reveals a further source of decay with a rate of $\approx 500 \mathrm{~s}^{-1}$ when the trap has a zero field minimum. We attribute this decay to transitions caused by interactions between neighboring Rydberg atoms and by fluctuating fields near the zero-field point of the trap.

The results presented herein demonstrate that Stark deceleration and off-axis trapping of Rydberg atoms enable one to suppress collisional losses, to study trap-loss mechanisms over extended periods of time, and opens an attractive route toward continuous trap-loading schemes.

This work is supported by the Swiss National Science Foundation (Project No. 200020-132688) and the European Research Council advanced grant program (Project No. 228286).

[1] M. T. Bell and T. P. Softley, Mol. Phys. 107, 99 (2009).

[2] L. D. Carr, D. DeMille, R. V.Krems, and J. Ye, New J. Phys. 11, 055049 (2009).

[3] S. Y. T. van de Meerakker, H.L. Bethlem, and G. Meijer, Nature Phys. 4, 595 (2008).

[4] B. Friedrich and J. M. Doyle, Chem. Phys. Chem. 10, 604 (2009).

[5] H. L. Bethlem, G. Berden, and G. Meijer, Phys. Rev. Lett. 83, 1558 (1999).

[6] N. Vanhaecke, U. Meier, M. Andrist, B.H.Meier, and F. Merkt, Phys. Rev. A 75, 031402(R) (2007).

[7] S. D. Hogan, D. Sprecher, M. Andrist, N. Vanhaecke, and F. Merkt, Phys. Rev. A 76, 023412 (2007).

[8] E. Narevicius, A. Libson, C. G. Parthey, I. Chavez, J. Narevicius, U. Even, and M. G. Raizen, Phys. Rev. Lett. 100, 093003 (2008).

[9] S. R. Procter, Y. Yamakita, F. Merkt, and T. P. Softley, Chem. Phys. Lett. 374, 667 (2003).

[10] E. Vliegen, S. D. Hogan, H. Schmutz, and F. Merkt, Phys. Rev. A 76, 023405 (2007).

[11] S. D. Hogan, Ch. Seiler, and F. Merkt, Phys. Rev. Lett. 103, 123001 (2009).

[12] P. F. Barker and M. N. Shneider, Phys. Rev. A 66, 065402 (2002).

[13] A. Osterwalder, S. A. Meek, G. Hammer, H. Haak, and G. Meijer, Phys. Rev. A 81, 051401(R) (2010).

[14] S. A. Meek, H. Conrad, and G. Meijer, Science 324, 1699 (2009).

[15] S. Y.T. van de Meerakker, R. T. Jongma, H. L.Bethlem, and G. Meijer, Phys. Rev. A 64, 041401(R) (2001).

[16] S. D. Hogan and F. Merkt, Phys. Rev. Lett. 100, 043001 (2008).

[17] E. Vliegen and F. Merkt, J. Phys. B 39, L241 (2006).

[18] E. Vliegen, P. A.Limacher, and F. Merkt, Eur. Phys. J. D 40, 73 (2006).

[19] H.A. Bethe and E.E. Salpeter, Quantum Mechanics of One- and Two-Electron Atoms (Springer, Berlin, 1957).

[20] T.F. Gallagher, Rydberg Atoms (Cambridge University Press, Cambridge, England, 1994).

[21] W. P. Spencer, A. G. Vaidyanathan, D. Kleppner, and T. W. Ducas, Phys. Rev. A 26, 1490 (1982).

[22] I. I. Beterov, D. B. Tretyakov, I. I. Ryabtsev, V. M. Entin, A. Ekers, and N. N. Bezuglov, New J. Phys. 11, 013052 (2009).

[23] A. A. Kamenski and V. D. Ovsiannikov, Can. J. Phys. 81, 755 (2003).

[24] I. L. Glukhov, E. A.Nekipelov, and V.D. Ovsiannikov, J. Phys. B 43, 125002 (2010).

[25] M. N. R. Ashfold, G. A. King, M. G. D. Nix, and T. A. A. Oliver, in Handbook of High-Resolution Spectroscopy, edited by M. Quack and F. Merkt (John Wiley \& Sons, Chichester, 2011). 